\title{
DIDATTICA TEATRALE E APRENDIMENTO DELL'ITALIANO COME LINGUA STRANIERA
}

\author{
LEARNING ITALIAN AS A FOREIGN LANGUAGE TROUGH DRAMA
}

Alessandra De Martino

Università di Warwick

\section{Riassunto:}

La didattica teatrale, impiegata in variegate attività nel campo dell'apprendimento, migliora le capacità comunicative. Sebbene non esclusivamente, questa tecnica è particolarmente efficace nell'insegnamento dell'italiano e delle tecniche di traduzione agli stranieri, permettendo l'esplorazione della lingua da parte del docente e del discente e realizzandone lo scopo comunicativo, come illustrano i casi e $\mathrm{i}$ metodi impiegati con studenti universitari.

\section{Parole chiave:}

Didattica teatrale, drammatizzazione, italiano.

glottodidattica,

\section{Abstract:}

Educational drama refers to a wide range of activities in an educational context improving communicative skills. Although not exclusively, it is within language and translation teaching that such a technique is exploited at its best, as it allows both teacher and learner to explore the language in depth and thus fulfil its communicative aim. This chapter discusses case studies and methods employed with undergraduate students.

\section{KeYwORDS:}

Educational drama, italian, glottodidactics. 


\section{PREMESSA}

In questo capitolo illustrerò la mia esperienza triennale di didattica teatrale nell'insegnamento dell'italiano come lingua straniera a studenti universitari di diverse nazionalità, tra cui anche alcuni madrelingua italiani. L'impiego del mezzo teatrale si è rivelato molto efficace in quanto ha permesso agli studenti di apprendere la lingua in modo creativo, superando i normali ostacoli che si incontrano durante il processo di apprendimento, e in maniera rilassata ed empatica, sviluppando un'intesa e una collaborazione che sarebbe stato più difficile sviluppare con un metodo puramente linguistico. Al piacere di imparare da parte degli studenti, si è affiancato il piacere di insegnare e di facilitare l'apprendimento della lingua e della cultura italiana, nella convinzione che il teatro sia uno strumento importante con il quale, attraverso la comune esperienza degli attori/ttrici e del pubblico, si può contribuire a cambiare la società.

\section{INTRODUZIONE}

Nel nostro mondo sempre più connesso e interdipendente a livello globale, la comunicazione è alla base di tutto e, sebbene stia diventando sempre più di tipo visivo, le parole giocano ancora un ruolo fondamentale nella trasmissione dei contenuti di una cultura, e per farlo in maniera "densa"1 è necessario che la lingua sia contestualizzata, per realizzare la funzione del linguaggio quale attività sociale. Infatti, come affermano M. Byram e M. Fleming la lingua "è un mezzo di comunicazione e interazione con persone di un'altra società e un'altra cultura" (Byram e Fleming, 2010: 1-2). ${ }^{2}$ L'importanza del contesto in cui la lingua si pone è stato sottolineato da teorici dell'apprendimento e filosofi, tra cui lo psicologo russo Lev Vygotsky che ha sottolineato la natura sociale del linguaggio, e lo psicologo americano Jerome Bruner, dal cui pensiero prese l'avvio la teoria costruttivista dell'apprendimento, secondo la quale "la conoscenza è il frutto della elaborazione personale di ciascun individuo, per cui gli esseri umani non assorbono le nozioni come spugne ma elaborano la realtà dandole un preciso significato" (Wagner, 1998:16) ${ }^{3}$

1 Il termine "densa" traduce quello inglese "thick", impiegato dall'antropologo americano Clifford Geertz che, seguendo il pensiero di Gilbert Ryle, ha sottolineato l'importanza di una "descrizione densa" della cultura, la quale viene definita come "il contesto a cui possono essere attribuiti accadimenti sociali, comportamenti, istituzioni o procedimenti". Geertz, C., The Interpretation of Cultures, New York, Basic Books, Inc., Publishers, 1972, p. 250.

2 Si veda sul punto anche la panoramica di M. Magnani, "Il teatro nella glottodidattica: un'esperienza tra teoria e prassi", Internet. 6-6-2016. http://www.ledonline.it/linguae/allegati/ linguae0202magnani.pdf p. 50.

3 Vedremo più avanti che ciò avviene anche quando gli studenti partecipano ad attività di didattica teatrale . 
Dei tre approcci teoretici per l'apprendimento della lingua, quello comportamentale elaborato da Skinner, l'approccio innatista che si rifà alla Grammatica universale di Chomsky, è quello interazionista (Stinson e Winston, 2011: 480) che sembra meglio riflettere i principi del costruttivismo linguistico, in quanto sottolinea l'importanza dell' interazione sociale cheèalla base dell'apprendimentolinguistico.Questo approccio, che si basa sulle teorie socio-linguistiche sviluppatesi negli anni '80 e '90 nel mondo anglosassone, si associa spesso al cosiddetto Approccio Comunicativo (Communicative Language Teaching) formalizzato da Michael Canale e Merryll Swain nel 1980 (Canale e Swain, 1980), che considera l'insegnamento della lingua straniera funzionale alla comunicazione del/la discente, che diviene il fulcro del processo didattico, mentre all'insegnante spetta il compito di assistenza e di somministrazione degli strumenti per l'apprendimento. L'approccio comunicativo, quindi, è quello che meglio favorisce l'apprendimento di una lingua funzionale allo scambio interpersonale, in quanto rende il/la discente consapevole sia della propria cultura che di quella straniera, e crea una certa identificazione con la cultura altra (Byram e Fleming, 1998:4- 5).

Un altro elemento da tenere presente nell'apprendimento della lingua straniera è che la capacità di comunicare e gli elementi affettivi ${ }^{4}$ sono aspetti fondamentali, come affermato da Stephen Krashen e Jim Cummins che hanno studiato lo sviluppo dell'apprendimento di una seconda lingua. Gli studiosi sostengono che nell'apprendimento della L2 l'inconscio rappresenta il solo modo per raggiungere la scioltezza linguistica. "L'abbattimento dei freni affettivi che impediscono l'apprendimento della lingua, si può raggiungere attraverso compiti linguistici comunicativi contestualizzati e poco impegnativi a livello dell'apprendimento", tra cui rientra anche lo strumento teatrale (Pross, 1986: 36). Infatti, il mezzo teatrale ha il merito di presentare la lingua contestualizzata, il cui apprendimento sintattico e grammaticale avviene in maniera spontanea e a livello di subconscio. Inoltre, attraverso l'immedesimazione nel personaggio, il/la discente acquista un atteggiamento più distaccato verso se stesso/a, per cui il suo apprendimento avviene in maniera, per così dire filtrata, dalla finzione teatrale. Le barriere affettive, quindi, si riducono sensibilmente, dando luogo ad un assorbimento delle nozioni linguistiche contestualizzate e liberate dal loro peso didattico.

L'uso del teatro nella didattica, ovvero la didattica teatrale, fa parte da anni dei curricula scolastici delle scuole in Gran Bretagna. Si deve a Dorothy Heathcote, l'educatrice e docente universitaria inglese, originaria del West Yorkshire, l'avere impiegato per la prima volta negli anni '50 il teatro nell'insegnamento, precorrendo di quasi mezzo secolo l'approccio denominato "educational drama" o "drama

4 Tra gli elementi affettivi rientra per esempio la reticenza ad esprimersi per paura di commettere errori, comune tra coloro che apprendono una lingua straniera. 
in education", una metodologia che si sarebbe diffusa negli Stati Uniti, Canada, Australia, Europa, Israele e Sudafrica (Wagner, 1999:2). La didattica teatrale venne concepita come strumento per apprendere attraverso il teatro, guardando "la realtà attraverso la fantasia, per individuare il significato sottostante l'aspetto superficiale delle cose" (Wagner , 1998: 8). Questa metodología viene applicata all'insegnamento delle lingue straniere in Gran Bretagna dagli anni '90 (Schewe e Shaw, 1993) e solo quest'anno il MIUR (Ministero dell'Istruzione dell'Università e della Ricerca) ha inserito il teatro nella scuola italiana tra le discipline previste. Prima di considerare l'applicazione di questa metodología riguardo all'apprendimento dell'italiano come seconda lingua, soffermiamoci per un momento sull'obbiettivo principale della didattica teatrale che è quello di “creare un'esperienza attraverso la quale gli studenti possano comprendere le relazioni umane, empatizzare con le persone e interiorizzare i punti di vista alternativi ai propri" (Wagner, 1998: 5). Se consideriamo adesso l'apprendimento della lingua straniera, vediamo che il teatro attiva quei processi cognitivi descritti per l'apprendimento della lingua in quanto elimina i filtri affettivi (Pross, 1986: 37). In secondo luogo, esso sviluppa il ragionamento critico, che viene definito come quel processo che "comporta un'attività ragionata riguardo a situazioni problematiche che richiedono una riflessione o un intervento, in cui la persona fa delle valutazioni intellettive di qualità" (Bailin, 1998: 146). E ciò è precisamente quanto accade ai/lle discenti quando devono risolvere le problematiche dei personaggi e fornire un giudizio ragionato delle scelte alla base dell'interpretazione di un testo. Tra i compiti interpretativi si possono annoverare l'analisi psicologica dei personaggi o la drammatizzazione di un testo letterario, mentre decisioni di tipo pratico sono ad esempio l'udibilità a fini comunicativi, lavorando quindi sulla produzione orale (Bailin , 1998: 148). È importante ribadire che le scelte drammatiche vanno fatte tenendo conto del contesto in cui si svolge l'azione, e richiedono un adeguamento della lingua allo scopo drammaturgico specifico, quale la recitabilità.

La didattica teatrale pone quindi il/la discente al centro del proceso di apprendimento, che avviene in maniera costruttiva e non deduttiva, ed è di tipo olistico, in quanto coinvolge la persona nel suo insieme, sollecitandone l'empatia, l'immedesimazione e la condivisione culturale della lingua straniera, mentre gli insegnanti agiscono da facilitatori, e non da somministratori di sapere. Per quanto riguarda le abilità linguistiche, la didattica teatrale facilita la produzione orale poiché gli studenti sono portati ad affrontare registri e stili linguistici diversi e ad ampliare il vocabolario usato generalmente in classe. Inoltre, essi aumentano la scioltezza di espressione e la comprensione nella lettura. Un'altro beneficio della didattica teatrale è il miglioramento della produzione scritta in quanto gli studenti, mentre sono stimolati a considerare diversi punti di vista e a usare il simbolismo come strumento di scrittura, aumentano la loro percezione dell'altro, ovvero gli spettatori (Wagner, 1998: 135). Vedremo 
più avanti, nell'analisi della drammatizzazione del racconto di Gianrico Carofiglio, Giulia, che gli studenti hanno realizzato tutti i fattori appena evidenziati, creando una breve pièce teatrale di enorme impatto empatico, combinando elementi testuali, immaginativi, simbolici e vocali. Da quanto appena esposto, deriva che attraverso il teatro, si può attuare un approccio linguistico "transculturale" e si creano "parlanti interculturali" ovvero persone che attraverso la lingua, vengono a conoscenza di una o più culture o identità e realizzano quindi una forma di "teatro interculturale" che ha come mezzo la lingua straniera (Byram e Fleming, 2010: 8-9). Questo genere di teatro, mirante al superamento dei pregiudizi derivanti dall'ignoranza della cultura altra, si rifà all'attività di ricerca svolta da Peter Brook che nel 1971 creò un gruppo di teatro interculturale sperimentale a Parigi che definì "the Culture of Links", la cultura dei legami. L'esempio di Brook è stato seguito anche da Ruth Handlen, una regista inglese che ha diretto in Francia nel 1994 Fragments of Clay ${ }^{5}$ servendosi della collaborazione di un insegnante di inglese francese. Sebbene la scioltezza linguistica non fosse l'obbiettivo primario, la performance fu di elevata qualità linguistica (Schmidt, 2010: 194). Da quanto esposto, possiamo affermare, in via preliminare, che fare teatro in una lingua straniera rende gli studenti consapevoli di altre culture, sviluppa una lingua immaginativa comune e aumenta l'autostima dei discenti attraverso la messinscena.

\section{DACIA MARAINI: PER PROTEGGERTI MEGLIO, FIGLIA MIA}

Fatta questa breve e, per necessità, sommaria introduzione teorica della metodologia, illustrerò la loro applicazione, attraverso la mia esperienza di docente di lingua italiana e tecniche di traduzione agli studenti del corso avanzato e superavanzato dell'Università di Warwick. Il primo esempio riguarda una traduzione collettiva dall'italiano in inglese del testo teatrale di Dacia Maraini Per proteggerti meglio, figlia mia. ${ }^{6}$ Il corso di lingua italiana include anche la traduzione da e verso l'inglese. Nelle ore di lingua abbiamo affrontato il tema della violenza domestica che ha trovato il suo complemento nell'ora di traduzione. L'utilizzo del mezzo teatrale mi è sembrato il più adatto per affrontare una tematica scottante, in quanto il filtro drammatico puntava l'attenzione su un soggetto diverso dal/la discente. I partecipanti includevano due ragazze spagnole, una francese, uno italiano e vari studenti inglesi. Nonstante la relativa vicinanza culturale e sociale, ovvero tutti ragazzi europei di classe borghese, durante il corso sono emerse molteplici

5 La pièce affrontava problematiche quali "la disoccupazione, i senzatetto, il divario tra ricchi e poveri e il "Sistema" che distrugge l'individuo". Schmidt, P., "Intercultural theatre through a foreign language", Ibi., p. 199.

6 Il titolo inglese è All the better to protect you, my dear. Il testo, nella forma di un doppio monologo, è stato commissionato a Dacia Maraini nel 2006 nell'ambito del progetto "Teatro per l'UNICEF" [per fare riflettere] sul termine "protezione" una delle parole che riassumono la Convenzione Internazionale delle Nazioni Unite sui diritti per l'infanzia e l'adolescenza". Maraini, D., Per proteggerti meglio, figlia mia, Roma, Giulio Perrone Editore, 2013, p. 49. 
diversità di vedute e soprattutto una diversa concezione del mondo, rispetto a quella italiana, oggetto del corso. È quindi interessante osservare come le problematiche sollevate dal testo abbiano stimolato negli studenti un'analisi critica e una presa di coscienza, abbiano generato un'empatia nei confronti dei personaggi, scaturita dalla discussione sui temi, e sulla lingua che veniva utilizzata. Questo è il metodo con il quale si è proceduto:

1. Gli studenti hanno letto il testo individualmente e poi abbiamo discusso in sede plenaria in classe le tematiche e gli aspetti linguistici più problematici.

2. Successivamente, gli studenti hanno tradotto sezioni del testo senza distinzione tra i personaggi, per cui alcune ragazze hanno tradotto sezioni della parte del padre e alcuni ragazzi hanno tradotto sezioni destinate alla bambina.

La prima versione era piuttosto letterale e abbiamo identificato vari errori di interpretazione che ho sottolineato, guidando gli studenti verso la correzione e verso traduzioni meno addomesticate, preferendo quindi il termine brioche piuttosto che bun; Signor invece di Mr.

Gli studenti si sono appassionati al testo e si è svolta una estesa discussione sul significato sottostante il testo, durante la quale alcuni di essi hanno proposto in maniera convinta le loro scelte traduttive. Ad esempio "gli echi si sono fatti più profondi" è stato tradotto con "the silence is louder now", sostituendo un'espressione sonora con un ossimoro silenzioso, oppure la frase "gli occhi si fanno cupi" è stata tradotta con "his eyes narrow", spostando l'accento dalla cupezza dello sguardo all'assottigliamento fisico degli occhi.

Il mio ruolo era principalmente quello di guida e di mediazione delle scelte traduttive, portando gli studenti verso soluzioni che tenessero conto anche delle esigenze della recitazione.

3. Dopo la fase iniziale, ho poi controllato il lavoro svolto e unito le varie parti, e insieme agli studenti abbiamo individuato ulteriori eventuali errori.

4. Gli studenti dovevano identificare problemi di coerenza, come ad esempio nell' uso del termine dad o daddy; mum o mummy; ${ }^{7}$ accuratezza, poiché dovevano tenere presente che cambiare un pronome o una preposizione avrebbe cambiano il significato dell'originale; recitabilità, ovvero dovevano preferire termini facilmente recitabili ad altri piu letterari.

5. Infine gli studenti hanno letto il testo alternandosi nelle varie sezioni per controllarne la scorrevolezza.

7 Dato che gli studenti avevano tradotto le singole sezioni indipendentemente, avevano usato $\mathrm{i}$ termini in maniera non omogenea. 
È interessante notare che, essendo gli studenti tutti del primo anno, non si conoscevano tra loro, ma attraverso la lettura del testo hanno sviluppato una maggiore confidenza; inoltre, nel momento della revisione incrociata del testo, fatta in maniera anonima, non essendo le varie versioni firmate, gli studenti hanno sviluppato anche la capacità di individuare errori e inesattezze, e proporre alternative. Quindi, questa attività, da un lato ha facilitato l'empatia degli studenti verso i personaggi, e dall'altro ha migliorato la loro capacità critica. Il lavoro è stato rappresentato, come lettura drammatica, al Warwick Student Arts Festival nel 2014 da studenti della Scuola di teatro dell'università. ${ }^{8}$

Lo stesso testo è stato utilizzato per un'altra attività, questa volta strettamente linguistica, ovvero per l'approfondimento lessicale e per il miglioramento della pronuncia e dell'intonazione. Hanno preso parte quattro studenti, di cui una italiana madrelingua, una di origini italiane ma non madrelingua, una franco-spagnola e uno inglese. In preparazione alla lettura drammatica ho condotto un laboratorio che comprendeva esercizi di tecniche teatrali e linguistiche. Gli studenti, disposti in circolo, hanno fatto degli esercizi di respirazione e di rilassamento muscolare. Hanno praticato le vocali con un esercizio di "comunicazione cieca", hanno recitato degli scioglilingua e fatto esercizi di pronuncia sulle consonanti più difficili da pronunciare, tenendo conto delle problematiche specifiche per le diverse lingue di origine. Hanno fatto anche esercizi miranti a stabilire un contatto col pubblico e a sostenere la presenza scenica. Infine abbiamo letto il testo alla luce degli esercizi fatti durante il laboratorio. È importante precisare che l'obbiettivo principale non era di tipo performativo-teatrale ma linguistico, quindi, quando gli studenti hanno effettuato la lettura drammatica di fronte ai colleghi di altri corsi, l'accento non era sulla performance artistica, ma sulla capacità di comunicare la cultura altra attraverso il mezzo teatrale. Lo studente aveva il ruolo del padre e le tre studentesse si alternavano nella parte della figlia. Durante la lettura i problemi di pronuncia e di intonazione erano stati in gran parte risolti. Alla lettura è seguita una discussione con numerose domande che vertevano principalmente sulla comprensione e resa del testo e sull'utilità del teatro per apprendere la lingua e per migliorare la propria sicurezza nella comunicazione. È interessante notare che, sebbene prima della lettura gli studenti fossero tesi, dopo la performance, nel rispondere alle domande, avevano acquisito maggiore disinvoltura, dimostrata anche dalla loro postura decisamente rilassata. Gli studenti hanno apprezzato l'utilità dello strumento teatrale per approfondire l'argomento e per apprendere la lingua, e hanno beneficiato della possibilità di scandagliare la natura dei personaggi con le loro problematiche.

\section{GIANRICO CAROFIGLIO: GIULIA}

8 Il filmato è disponibile sul sito dell'International Network of Italian Theatre al seguente indirizzo: http://www.init.org.uk/performances-2 
Attraverso i casi descritti abbiamo visto che il teatroè un mezzo efficace per sviluppare competenze linguistiche orali e traduttive. I prossimi esempi illustrano l'impiego della didattica teatrale per il miglioramento delle capacità di scrittura. Tra i testi utilizzati nel corso di lingua, è inclusa anche una raccolta di racconti brevi intitolata Non esiste saggezza, di Gianrico Carofiglio. Ho scelto questo testo perché le tematiche affrontate sono di grande attualità e offrono spunti di discussione stimolanti, e perché si presta anche a varie attività di scrittura. Inoltre, lo stile in cui è scrittoè scorrevolee nel contempo raffinato e accattivante. Il testo, scelto dagli studenti, è stato utilizzato per un'attività di scrittura creativa, consistente nella drammatizzazione del racconto Giulia. Tre studenti, una madrelingua e due con competenze linguistiche molto avanzate, ma per i quali l'italiano è L2, hanno trasposto il racconto, scritto in prima persona, in un testo per tre personaggi: una bambina, uno psichiatra e una giornalista radiofonica che forniva il retroscena dei fatti. Il racconto narra la storia di una bambina di circa otto anni, Giulia, che viene uccisa da un pedofilo appartenente all'alta borghesia barese. Il protagonista, uno psichiatra infantile, la incontra, inspiegabilmente, prima della sua morte, che scopre poi essere avvenuta l'anno prima, quando l'accompagna (o l'ha accompagnata?) all'indirizzo del suo assassino senza saperlo. Il racconto, surreale, giustappone passato e presente lasciando al lettore e alla lettrice l'interpretazione finale dei fatti. Nella drammatizzazione lo sviluppo luogo-temporale è stato compresso e riflette il principio drammaturgico del "qui-ora". 9 La lettura è stata integrata da una canzone, scritta, cantata e registrata dalla studentessa madrelingua, che rielaborava brani del testo originale in forma poetica e faceva da intermezzo o sottofondo in alcuni momenti della pièce. Anche in questo caso la performance consisteva in una lettura drammatica, ma sono stati aggiunti alcuni oggetti di scena, quali una radio e una borsetta, che gli studenti hanno usato durante la recitazione. Inoltre, l'inserimento della registrazione ha richiesto ulteriore confronto, coordinamento e intesa. Essendo la competenza linguistica degli studenti molto avanzata, il laboratorio linguistico-teatrale condotto in preparazione della lettura si è concentrato soprattutto sulle tecniche di respirazione e sugli esercizi di intonazione. La risposta affettiva degli studenti è stata di notevole intensità, a causa della tematica che ha richiesto l'impiego di strategie di scrittura, approfondimento e ricerca del significato di termini anche da parte della studentessa madrelingua. Vale la pena notare, a questo proposito, che questa metodologia si è rivelata utile anche per l'apprendimento e il miglioramento dell'italiano come L1, in quanto ha generato una riflessione sulla lingua e sulla sintassi, nonché sul parlato, e ha affinato l'abilità di scrittura anche della parlante madrelingua. La lettura drammatica

9 Mi rifaccio alla classificazione di Patrizia Monaco fornita nel suo "Decalogo di drammaturgia", in cui uno degli elementi del testo drammatico è "l'Eterno presente, poiché sulla scena tutto avviene qui ed ora", contenuto nel presente volume. Un'altro elemento ritrovato nella drammatizzazione è quello della "Scenografia verbale, cioè evocare i luoghi con le parole", poiché la resa linguistica rappresentava efficacemente i luoghi della storia. 
di fronte a studenti e docenti ha riscosso un grande apprezzamento, e la recitazione ha avuto forte impatto emotivo ed efficacia drammatica sul pubblico.

\section{STEFANO BENNI: TORQUATO ABBANDONATO}

Il quarto e ultimo esempio ha ad oggetto un altro racconto, questa volta di Stefano Benni, dal titolo Torquato abbandonato. Il testo affronta, con lo stile ironico di Benni, l'argomento dell'abbandono degli anziani da parte dei parenti durante festività e vacanze estive, e racconta la storia di un nonno pensionato che viene lasciato solo a casa, dai familiari con cui vive, in un torrido Ferragosto, senza provviste alimentari nè alcun conoscente come punto di riferimento. Per procurarsi da mangiare, vaga per la citta deserta e assolata dove subisce anche atti di razzismo (viene scambiato per un marocchino a causa del turbante che si era confezionato con un lenzuolo bagnato per proteggersi dal caldo) e offese varie, e alla fine si rifugia nel garage del condominio in cui abita, dove si unisce a una schiera di coinquilini pensionati, anch'essi abbandonati, che nel corso degli anni avevano allestito uno stabilimento balneare cittadino con tanto di piscina e frigoriferi. Anche questa storia ha un finale aperto sulla sorte di Torquato: sarà morto di calore o è fuggito con la sua innamorata Ines del piano di sopra? Tre studentesse hanno scelto di fare l'adattamento teatrale del racconto, che peraltro aveva molti personaggi, rendendolo un monologo recitato a tre. ${ }^{10}$ Sebbene la loro competenza linguistica fosse elevata, nessuna di esse era madrelingua italiana, ${ }^{11}$ e la ulteriore difficoltà, in questo caso, risiedeva nel genere comico del monologo che doveva avere la stessa efficacia esilarante del racconto. Inoltre, doveva essere rispettata la regola del qui-ora, mentre il racconto si spostava in vari luoghi. Anche in questo caso si è reso necessario un ragionamento critico per valutare le tematiche e il carattere dei personaggi. Inoltre si è dovuto lavorare sulla struttura del monolgo che, per quanto dovesse fornire gli elementi essenziali della vicenda, doveva mantenere viva l'attenzione degli spettatori senza dilungarsi su aspetti narrativi del racconto che a livello drammatico avrebbero appesantito il ritmo della pièce. ${ }^{12}$ Le studentesse hanno lavorato separatamente alla drammatizzazione del testo, e si sono incontrate a Londra durante la pausa delle vacanze di Pasqua per discutere e coordinare le rispettive versioni del monologo, che sono state poi fuse in un unico testo coerente che mi hanno inviato per una prima verifica. Quando ci siamo incontrate, anche in questo caso il mio intervento consisteva principalmente nell'evidenziare le questioni lessicali

10 Sebbene la pièce fosse sostanzialmente un monologo, c'erano anche brevi interventi ad hoc di altri personaggi.

11 Una studentessa era di origine franco-italiana, una anglo-olandese e l'altra anglo-italiana, ma tutte nate e cresciute in Inghilterra

12 Un altro requisito del testo teatrale descritto da Patrizia Monaco è "[i]l Ritmo, che deve variare di continuo, come nella musica. Si scrive pertanto ad alta voce". 
e grammaticali; suggerire degli aggiustamenti del testo per renderlo più recitabile; dirigere gli esercizi di pronuncia e intonazione e dare indicazioni di regia per la lettura. Un elemento apprezzabile è stato anche l'inserimento di elementi culturali, quali la marca di un particolare caffè napoletano, o l'uso di espressioni idiomatiche. La lettura drammatica di fronte agli studenti e ai docenti è stata di notevole effetto comico e le tre studentesse hanno mostrato ottime capacità espressive e di comunicazione. Il fatto di esprimersi in italiano, mentre ha contribuito all'immedesimazione culturale delle studentesse, ha favorito una riflessione sul problema della società che invecchia anche nel loro paese di appartenza. Inoltre, attraverso la drammatizzazione, hanno impiegato e praticato un linguaggio contestualizzato e finalizzato alla comunicazione, e hanno avuto la possibilità di ricercare termini che esulavano da quelli normalmente impiegati durante le lezioni. ${ }^{13}$

Prima di concludere si rende necessaria una precisazione: sebbene il processo di apprendimento giocasse un ruolo fondamentale in tutte le attività che ho descritto, era importante che la resa performativa rappresentasse, sia per i discenti che gli spettatori, un prodotto di qualità. Infatti, nonostante ci sia una corrente di pensiero che predilige il processo rispetto al risultato, penso che quest'ultimo sia ugualmente importante per non rischiare di ridurre il tutto ad un esercizio fine a se stesso. ${ }^{14}$ La lettura drammatica, la drammatizzazione di un testo letterario e la traduzione di un testo teatrale rappresentano, quindi, un valido strumento per l'insegnamento di una lingua straniera, e per l'approfondimento della L1 da parte dei/lle discenti madrelingua, e per la pratica della scrittura creativa. Attraverso il teatro gli studenti hanno avuto l'opportunità di approfondire la lingua straniera liberandosi dai filtri affettivi, apprendendo la lingua in maniera contestualizzata. Affrontare le problematiche dei testi ha sviluppato la loro empatia e ha favorito la ricerca di una lingua comune che rispondesse ai criteri di comunicazione, favorendo un apprendimento critico del linguaggio e della cultura altra. Nel caso dell'adattamento teatrale dei racconti di Gianrico Carofiglio e di Stefano Benni, la drammatizzazione ha migliorato la valutazione e la riproduzione dei registri, sperimentando con testi sia drammatici che comici, mentre la lettura drammatizzata ha migliorato la pronuncia e l'intonazione sia nella L1 che nella L2. Inoltre, la lettura recitata ha migliorato l'autostima e la capacità di comunicazione. Per quanto riguarda la traduzione del testo di Dacia Maraini, essa ha consentito agli studenti di prendere

13 Una delle studentesse ha così commentato la sua esperienza: "La drammatizzazione di questo racconto mi ha dato molto. Il procedimento di trasformazione di un racconto in un monologo teatrale $\mathrm{mi}$ ha aiutato a sviluppare le mie capacità di lettura e interpretazione di testi italiani. Soprattutto credo che mi abbia aiutato a migliorare la pronuncia e mi ha infuso più sicurezza nel parlare italiano davanti a un pubblico".

14 Si veda sul punto Di Martino, E., Di Sabato, B., "Playing a Part: Drama in the Language Classroom", Studying Language through Literature, Newcastle upon Tyne, Cambridge Scholars Publishing, 2014. 
maggiore coscienza sia dell'italiano che dell'inglese e di approfondire il significato di parole nuove che esulano dal loro vocabolario.

Ho aperto questo capitolo con un riferimento alla realtà globalizzata in cui viviamo, che, sebbene comprenda culture diverse, rischia di imporre la visione di una società dominante sulle altre. Per evitare ciò è necessario mantenere un' ottica transnazionale e interculturale che permetta di conoscere l'altro per rapportarsi al sé, di beneficiare delle diversità e al tempo stesso di conservare la propria identità. Attraverso la conoscenza e la contestualizzazione delle lingue nelle rispettive culture si può realizzare una lettura "densa" di esse, e il teatro ce ne offre il modo.

\section{BIBLIOGRAFIA}

Bailin, S., "Critical Thinking and Drama Education", Research in Drama Education: The Journal of Applied Theatre and Performance, 3, (1998), pp. 145-153.

Benni, S., “Torquato abbandonato” il Venerdì di Repubblica, 13-8-2010, n. 1169.

Byram, M., Fleming, M., Language Learning in Intercultural Perspective. Approaches through drama and ethnography, Cambrdige, Cambridge University Press, 2010.

Canale M., Swain M., "Theoretical Bases of Communicative Approaches to Second Language Teaching and Testing", Applied Linguistics, 1 (1980), pp.1- 47. Internet. http://ibatefl.com/wp-content/uploads/2012/08/CLT-Canale-Swain.pdf Carofiglio,

G., "Giulia”, Non esiste saggezza, Milano, Rizzoli, 2010, pp. 57-68.

Di Martino, E., Di Sabato, B., "Playing a Part: Drama in the Language Classroom", Studying Language through Literature, Newcastle upon Tyne, Cambridge Scholars Publishing, 2014.

Geertz, C., The Interpretation of Cultures, New York, Basic Books, Inc., Publishers, 1972. Magnani, M., “Il teatro nella glottodidattica: un'esperienza tra teoria e prassi” Internet. 6-6-2016. http://www.ledonline.it/linguae/allegati/linguae0202magnani.pdf

Maraini, D., Per proteggerti meglio, figlia mia, Roma, Giulio Perrone Editore, 2013.

Pross, E. E. "Using Theatre Games to Enhance Second Language Acquisition in Bilingual Education", Bilinugal Review / La Revista Bilingüe, 13, (1986), pp. 35- 40.

Schewe, M., Shaw, P., a cura di, Towards Drama as a Method in the Foreign Language

Classroom, Frankfurt, Peter Lang, 1993.

Schmidt, P., "Intercultural theatre through a foreign language", Language Learning in Intercultural Perspective, Cambrdige, Cambridge University Press, 2010, pp. 193- 203.

Stinson, M., Winston, L., "Drama education and second language learning: a growing field of practice and research", Research in Drama Education: The Journal of Applied Theatre and Performance, 11 (2011), pp. 479-488. 
Internet. http://www.tandfonline.com/doi/abs/10.1080/13569783.2011.616395

Wagner, B. J. Educational Drama and Language Arts, Portsmooth, Hainemann, 1998.

------, "A Theoretical Framework for Educational Drama", Educational Drama, and Language Arts, Portsmooth, Hainemann, 1998, pp. 15-33.

------, Dorothy Heathcote. Drama as a Learning Medium, Portsmouth, Heinemann, 1999. 